Volume 8, No.1.4, 2019

International Journal of Advanced Trends in Computer Science and Engineering

Available Online at http://www.warse.org/IJATCSE/static/pdf/file/ijatcse2681.42019.pdf

https://doi.org/10.30534/ijatcse/2019/2681.42019

\title{
A Seamless Multi-Touch Interaction for Collocated Multi-Mobile System
}

\author{
Teo Rhun Ming ${ }^{1}$, Ong Beng Liang $^{2}$, Noris Mohd Norowi ${ }^{3}$, Rahmita Wirza O.K. Rahmat ${ }^{4}$, Azrul Hazri \\ Jantan $^{5}$ \\ Faculty of Computer Science and Information Technology, \\ Universiti Putra Malaysia, \\ 43400, Serdang, Selangor \\ rhmteo@outlook.com ${ }^{1}$,obl_0512@yahoo.com ${ }^{2}$, noris@upm.edu.my ${ }^{3}$,rahmita@upm.edu.my ${ }^{4}$, \\ azrulhazri@upm.edu.my ${ }^{5}$
}

\begin{abstract}
Collocated Multi-Mobile System allows users to combine mobile devices as a massive surface with multi-touch capabilities for collaborative activities. In this paper, a study was conducted to identify the performance of a seamless multi-touch interaction using multiple mobile devices. The system owned a server that used to unify touch-input from multiple mobile devices thus able to create large multi-touch surface. Trough user-centred approach, this study found that the seamless multi-touch interaction was effective and intuitive during interaction across multiple devices. There were less errors and less movements made by users while doing certain task using the seamless multi-touch interaction. Issues such as bezel and lack of gesture were considered and will be resolved in the future.
\end{abstract}

Key words : mobile devices; tabletop; multi-mobile system; collocated multi-mobile system; seamless multi-touch interaction.

\section{INTRODUCTION}

With the recent technology enhancement, there have a lot of research that studies of using the tabletop system as a collaborative tool for a group of users. The tabletop system allows a group of people to collaborate to accomplish a single target for information visualization [1]. However, most of the tabletop systems are not very portable and costly to own by regular users [1]. The study intents to overcome this issue by combining multiple mobile devices to form a collocated multimobile system. The collocated multi-mobile system provides users an alternative collaborative solution which is much affordable and portability. An early example of research was conducted by Lyons, Pering, Rosario, Sud, \& Want as they developed collocated multi-mobile system which combines multiple mobile tablets to study the effect of group collaboration on large interactive surface [2]. The collocated multi-mobile system has been used for collaboration purposed, such as presentation, discussion and entertainment.
This paper will study on the lack of seamless multi-touch interaction to manipulate an object using multi-touch interaction that was not limited to a single device but available to be used even users perform multi-touch on multiple device. The tabletop system able to perform all sort of gesture such as zooming using pinch, rotating clockwise and anti-clockwise, drag and drop anywhere on the surface [3]. Most of the collocated multi-mobile system available had limited multi-touch interaction to a single device. Performing multi-touch on collocated multi-mobile system was not straight forward like performing multi-touch at any surface on a tabletop system. Previous study on collocated multi-mobile system only allowed users to perform simple gesture like drag and drop across device [4]. However, the system did not provide users more natural multi-touch interaction on the object for spatially aware interaction [5].

Some collocated multi-mobile system can only recognize touch input only on single mobile device instead of multiple mobile device [6]. This study intent to propose an interactive collocated multi-mobile system by using multiple mobile device to support collaboration activities beyond device boundaries. Figure 1(a) shows the multi-touch interaction on a single device while Figure 1(b) shows seamless multi-touch interactivity that is not limited to a single mobile device but available at any surface of multiple mobile device.

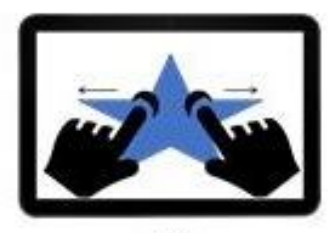

(a)

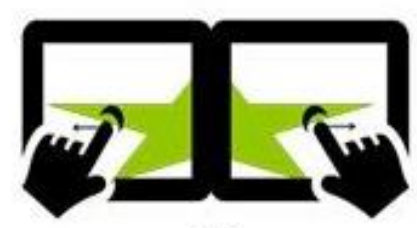

(b)
Figure 1: (a) Individual multi-touch interaction on a single device (b) Seamless multi-touch interaction on multiple mobile device. 


\section{RELATED WORKS}

There are a few researches that study on the user experience of seamless multi-touch interaction on collocated multimobile system. This study was more toward the spatialaware interaction so interaction with the content was more natural and familiar. Spatially aware interaction uses realworld spatial configuration as referential domain [5]. Spatial aware interaction such as drag and drop objects from device to device [7], flicking and throwing objects between phone to phone [8].

Most common interaction on collocated multi-mobile system was the cross-device interaction and synchronous gesture. The collocated multi-mobile system had been using cross device interaction as a common way to transfer information between mobile devices. Most of the collocated multi-mobile system use drag and drop interaction to transfer files or data across mobile devices. Pick-and-Drop had a feature that transfer an object by dragging and dropping across multiple mobile device [7]. Pick-and-Drop had a pen-based direct manipulation technique that was used for data transfer between multiple mobile devices. Stitch Master was able to transfer photo from one device to another. The interaction is very simple by touching and moving a photo across the boundaries of a device. On another device, the touch is release and the photo was transferred. Nevertheless, the limitation was that it was the only way of interacting with the object across multiple mobile device.

Some collocated multi-mobile systems were allowed to perform multi-touch interaction across multiple mobile device for specific function. The interaction used synchronous gesture that required users to perform synchronize action on multiple mobile device for specific task. Example of study that used synchronous gesture was Ohta \& Tanaka that used pinch gesture to align multiple tablets to form a composite display

[9]. To perform pinch gesture, user would first put one finger on one device and another finger on another device. Then, user would perform synchronous action like pinching to stitch the mobile device together. The synchronous gesture was mostly used on specific task and was not useful for spatially aware interaction.

Throughout the review on past researches, it was found that most of the multi-touch interaction on collocated multi-mobile system was limited only on a single device. Previous work done by Rädle et al. for HuddleLamp system has gestures such as pinch-to-zoom but it was limited within device boundaries

[6]. Currently, multi-touch interaction only able to perform on single device. The system failed to recognize multi-touch interaction that make used of the whole screen surface by putting one finger on one device and other fingers on other device. This study focuses on bringing seamless ways to interact the object with multi-touch that not confined to a single mobile device but on multiple mobile device.

\section{SYSTEM DESIGN}

The collocated multi-mobile system was built for the Android Operating System. The collocated multi-mobile system was built based on client-server architecture (Figure 2 ). When the application launched within the devices, they connected to the Wi-Fi network.

When touch-input happens anywhere on any one of the devices, that particular device sends the coordinate and event to the server. The server maps the coordinate of the device to the global coordinate. The event was also being determined on the server. The server then sends back the transformed coordinate to the client. The clients then render their part of the object in the coordinates sent by the server. The object was then transformed according to the location and orientation of the devices to create a sense of large display.

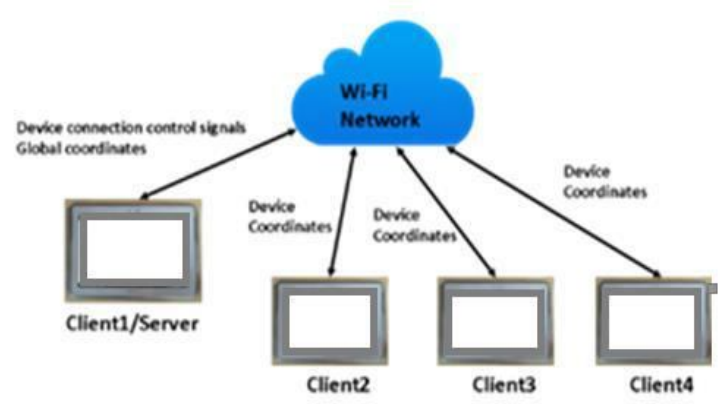

Figure 2: Server-Client Architecture in Collocated Multi-Mobile System

\section{EVALUATION}

\subsection{Participants}

Sixteen full-time undergraduate university students were voluntarily recruited for the study. The participants were ranged in age from 19 to 23 years $($ mean $=20.8$ years, $s d=$ 1.4 years). All of them spoke English as their second language, and all were fluent and proficient English speakers. All participants have a mobile phone and most of they use the phone for the Internet Surfing (100\%), Social Media (87.5\%), Education (81.25\%), Entertainment (75\%) and Services $(68.75 \%)$.

\subsection{Task Material}

The collocated multi-mobile devices system was originally developed as a preliminary study based on a simple interaction design lifecycle. The collocated multi-mobile system was developed for Android operating system. Two types of dragdrop application were developed for the study. Both applications were drag and drop applications where users could drag, rotate and scale on an image. The main difference between two applications was the one of them has the ability to perform multi-touch gesture across multiple devices. Figure 3 shows the drag and drop application used for collocated multi-mobile devices system. 

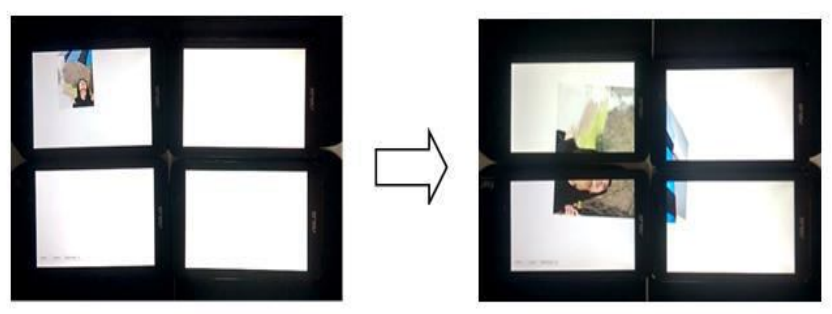

Figure 3: The drag and drop application for collocated multimobile system allows for objects to be drag drop and share across multiple mobile device

\subsection{User Study}

At the beginning of the evaluation session, all participants needed to sign a consent form. Then the main experimenter explained the study information and procedure to each group and they were allowed to ask questions on any issues regarding the study. Each participant was equipped with a pair of colored paper wristbands for easy recognition during analysis stage.

A simple training session was conducted as a warm-up session for users with the system. During the training session, the main experimenter explained the system functionality/features to the participants. Participants were allowed to play around/to get familiar on the connected multi-screen devices using image drag-and-drop application.

At the beginning of the evaluation session, one of the group members were asked to read the task instruction. During the session, participants were required to interact an image with multi-touch gestures. There were two types of drag-drop application, application A only had multi-touch gestures limited to a single mobile device and application B had seamless multi-touch interaction across multiple mobile device. Participant required to do image sharing task using application A and application B. First, participants were required to use application A to share and to interact photos with friends. Participants were required to drag the image to any of the tablet and then to zoom the image to double its original size. Then, the participants were asked rotate the image for about 180 degrees. Participants then needed to drag the image to the center and zoomed down the image to its original size. Lastly, rotate the image for about 180 degrees. The task was repeated for application B.

Upon completion of the task, a group face-to-face interview were conducted and moderated by the experimenter. All participants shared their experiences towards the evaluation task. After that, each participant completed the questionnaire; information included the participant's demographic background, the participant's current practice of collaborative activities preferences, awareness and natural of multi-touch gesture that goes beyond the boundaries of a device and interface review of the collocated multi-mobile system. All sessions were recorded with a video camera for analysis purpose. The video was analyzed where the number of gestures used in the border to do functional moves on the pieces to arrange the picture include dragging, scaling and rotating was noted.

\section{RESULTS}

The data was gathered and analyzed through observations, video recordings, questionnaires and interviews to observe the effectiveness of seamless multi-touch interaction on collocated multi-mobile system. There were a few aspects that focus for this user study:(1) to determine the effectiveness of seamless multi-touch interaction on collocated multi-mobile system; (2) to find out how intuitive and useful of seamless multi-touch interaction on collocated multi-mobile system; and (3) to find the workload its perceive by the users. The results of the study are presented in Figure 4 and Figure 5.

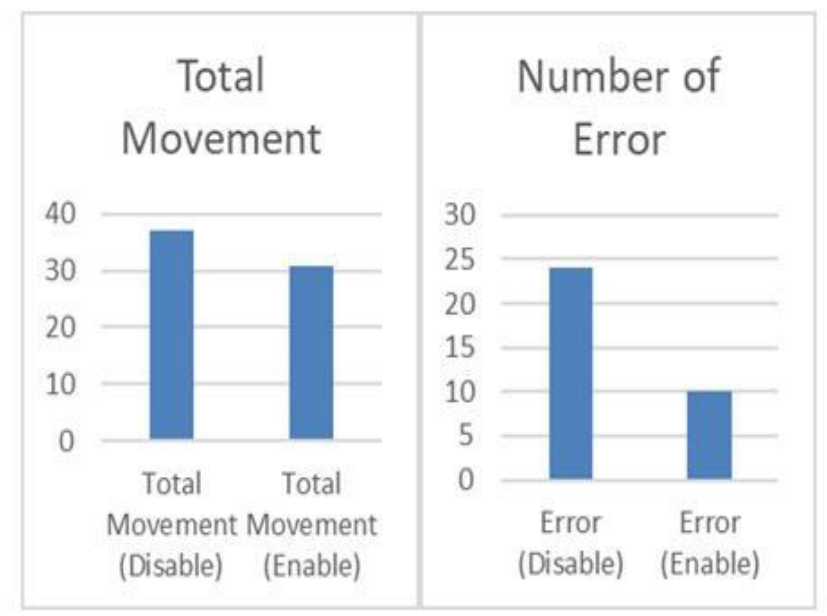

Figure 4: The total movement and number of errors between unify and individual multi-touch interaction

Figure 4 showed the total movement and number of error when seamless multi-touch interaction was disabled and enabled. The total movement for the disabled seamless multi-touch interaction (54.4\%) is higher than the enabled seamless multi-touch interaction (45.6\%). The result indicated that the seamless multi-touch interaction had helped to reduce a significant number of moves by eight percent $(8.8 \%)$. The number of errors made during the user study for disabled seamless multi-touch interaction is 24 (35.3\%). The number of errors produced from enabled seamless multi-touch interaction support is 10 (14.7\%) which is about 20.6 percent (20.6\%) of error reduction. Overall, the seamless multi-touch interaction enable the reduction in number of movement by $8 \%$ while also reducing the number of errors user made by $20.6 \%$. 


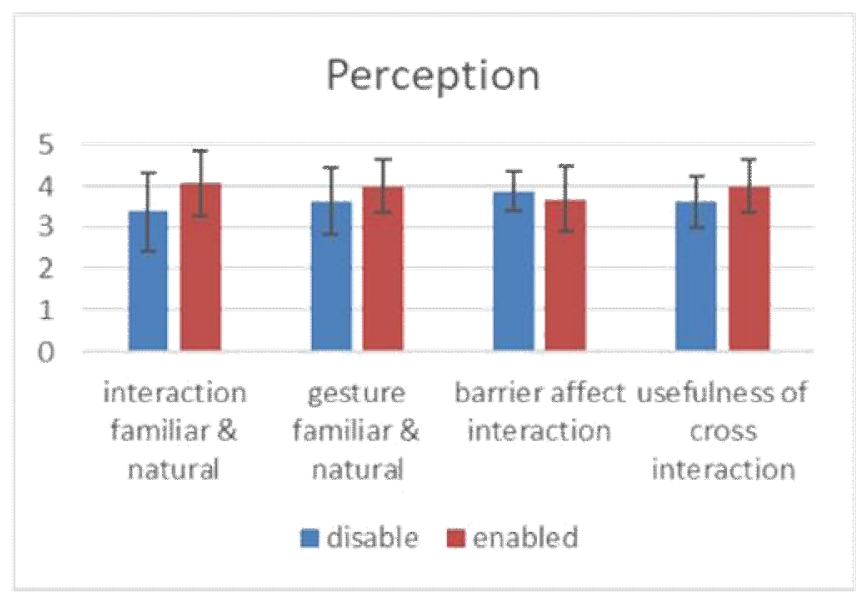

Figure 5: The result of questionnaire between unify and individual multi-touch interaction

For the questionnaire, the users required to answer some questions on the interaction intuitiveness and usefulness between disabled and enabled seamless multi-touch interaction. The users found that the system with enabled seamless multi-touch interaction was quite familiar and natural compared to disabled seamless multi-touch interaction ( $x=4.06$ versus $x=3.375)$. The gesture was also found to be much more familiar and natural for the enabled seamless multi-touch interaction compare to disabled seamless multi-touch interaction $(x=4.0$ versus $x=3.625)$. Overall, users found that the system with seamless multitouch interaction is quite useful $(x=4$ versus $x=3.625)$. However, the barrier between the display become obvious for enabled seamless multi-touch interaction compare to disabled seamless multi-touch interaction $(x=3.875$ versus $x=3.6875)$. This was because more user would like to perform gesture near the border as seamless multi-touch interaction was enabled but the border prevents continuous interaction.

During the interview, users were asked them how intuitive was the application with enabled seamless multi-touch interaction compare to disabled seamless multi-touch interaction. The users were really satisfied with the outcome from the application as they were able to carry out multi-touch gestures anywhere on different mobile devices. The users even commented that they could use more than two fingers to scale and rotate an object across the border of the display.

"I love that I am be able to perform multi-touch on multiple tablets because there is a lot of space to do like this or do like this doing on a tablet or multiple tablet for rotation and scale," (Group 4 User 3).

"It is very easy because we can touch with two fingers also can do it with three fingers across multiple tablets," (Group 3 User 2).

\section{DISCUSSION}

The results show that seamless multi-touch interaction performs better than multi-touch interaction limited to a single device for object interaction. The fact that the participants preferred seamless multi-touch interaction across displays suggests that it is an attractive interaction system for collocated multi-mobile system.

The seamless multi-touch interaction would be benefited collocated multi-mobile system that uses small screen size mobile devices. Performing multi-touch on small screen was impractical because there was not enough screen real estate

[10]. Instead of performing multi-touch that limited on a small screen, the seamless multi-touch interaction could enable users to perform multi-touch gesture across multiple mobile device with ease. Seamless multi-touch interaction could free up some space due the fingers not cover up valuable screen information on single device.

The seamless multi-touch interaction could be useful in "around-the-device" application. There are a few examples of applications that support the "around-the-device" such as Pass-Them-Around, the photo-sharing application [11], MobiTop, the collaborative application [12] and MagMobile, the game application [13]. The seamless multi-touch interaction was useful for multi-touch on multi-device interaction especially on collocated multi-mobile application.

The seamless multi-touch interaction can also be useful for users who are collocated to perform a cooperative gesture [14]. For example, to enlarge an image, a user would perform dragging from one device, and then other users could cooperative expand the image by dragging the image at the opposite direction. This study only develops a drag and drop application, with the aim to demonstrate various uses of collocated multiple mobile device environment.

However, there are several areas need to be improved for the seamless multi-touch interaction in collocated multi-mobile system such as limiting the image size to overcome bezel/border issues and incorporating additional gesture feature to the seamless multi-touch interaction. Users found it difficult to perform interaction when image was smaller. To resolve this bezel issue, the application should limit the size of the object to be able to scale so that the object able to perform interaction near the bezel. Users also suggested to add an additional number of gesture such as flicking to move image from one device to another and double tap to return image to its original size. One interesting finding during the experiment was that users sometime tried to split to duplicate the image when using individual multi-touch setting. This ripping gesture could also can be implemented into the system for image duplication feature. 


\section{CONCLUSION AND FUTURE WORK}

In this paper, the implementation and approach to seamless multi-touch interaction so that multi-touch can work anywhere even there is a border between the devices and does not limited to a single device was described. It has been demonstrated that the seamless multi-touch interaction for collocated multi-mobile system is effective, useful and intuitive. It has also been explained that the seamless multitouch interaction can be quite useful for "all-around-device" applications. The issues and suggestion from the user study has been will be taken into account for improvement for future works. We convince that seamless multi-touch interaction able to be used for future cross-device interaction applications to support group collaborations.

\section{ACKNOWLEDGEMENTS}

This study is funded by the Geran Putra Berimpak grant (9549600), which the authors are grateful for. The authors would also like to thank all the study participants for their time and valuable feedback.

\section{REFERENCES}

1. L. Yee Lien, C. Soon Nyean, and Y. Wen Jiun, Enhanced Collocated Collaborative Learning of Bahasa Malaysia through Multi-touch Educational Application on Cost Effective Tabletop, Proceedings of the Int. Conf. Environ. Econ. Energy, Devices, Syst. Commun. Comput. Math., (2011), pp. 35-40.

2. K. Lyons, T. Pering, B. Rosario, S. Sud, and R. Want, Multi-display composition: Supporting display sharing for collocated mobile devices, Lect. Notes Comput. Sci. (including Subser. Lect. Notes Artif. Intell. Lect. Notes Bioinformatics), (2009), vol. 5726 LNCS, no. PART 1, pp. 758-771. https://doi.org/10.1007/978-3-642-03655-2_83

3. S. Kim, J. Kim, and C. Lee, Implementation of Multi-touch Tabletop Display for HCI (Human Computer Interaction), Techniques, (2007), pp. 854863.

4. E. Kurdyukova, M. Redlin, and E. André, Studying User-defined iPad Gestures for Interaction in Multidisplay Environment, Proceedings of the 2012 ACM Int. Conf. Intell. User Interfaces , (2012), pp. 93-96.

5. R. Rädle, H. Jetter, M. Schreiner, Z. Lu, H. Reiterer, Y. Rogers, R. Raedle, M. Schreiner, and H. R. De,

"Spatially-aware or Spatially-agnostic? Elicitation and Evaluation of User-Defined Cross-Device Interactions," Proc. CHI'15, (2015), pp. 3913-3922.

6. R. Rädle, H.-C. Jetter, N. Marquardt, H. Reiterer, and Y. Rogers, HuddleLamp, Proceedings of the 9th ACM Int. Conf. Interact. Tabletops Surfaces - ITS '14 , (2014), pp. 45-54.

https://doi.org/10.1145/2669485.2669500
7. J. Rekimoto, Pick-and-drop, Proceedings of the 10th Annu. ACM Symp. User interface Softw. Technol. - UIST '97, (1997), pp. 31-39. https://doi.org/10.1023/A:1000396603851

8. A. Lucero, J. Holopainen, and T. Jokela, "MobiComics," Proc. 14th Int. Conf. Human-computer Interact. with Mob. devices Serv. - MobileHCI'12 , (2012), p. 383. https://doi.org/10.1145/2371574.2371634

9. T. Ohta and J. Tanaka, Pinch: An interface that relates applications on multiple touch-screen by 'pinching' gesture, Lect. Notes Comput. Sci. (including Subser. Lect. Notes Artif. Intell. Lect. Notes Bioinformatics), (2012), vol. 7624 LNCS, pp. 320-335. https://doi.org/10.1007/978-3-642-34292-9_23

10. A. Butler, S. Izadi, and S. Hodges, "SideSight : Multi- • touch ' interaction around small devices," UIST '08 Proceedings 21 st Annu. ACM Symp. User interface Softw. Technol., (2008), vol. 23, no. 21, pp. 201-204.

11. A. Lucero, J. Holopainen, and T. Jokela, Pass-themaround, Proceedings of the 2011 annual conference on Human factors in computing systems - CHI '11, (2011), pp. 1787-1796. https://doi.org/10.1145/1978942.1979201

12. Beng Liang, O., Rhun Ming, T., Mohd Norowi, N., \& Hazri Jantan, A. (2018). Using Continuous Spatial Configuration for Bezel Issues in a Multi-Mobile System. International Journal of Engineering \& Technology, 7(4.31), pp. 431-436.

13. D. Y. Huang, T. W. Chang, M. L. Tsai, C. P. Lin, N. H. Yu, M. Y. Chen, Y. P. Hung, and C. H. Hsu, MagMobile:

Enhancing Social Interactions with Rapid ViewStitching Games of Mobile Devices, Smart Innov. Syst. Technol. , (2013), vol. 21, pp. 477-486. https://doi.org/10.1007/978-3-642-35473-1_48

14. K. Hinckley, G. Ramos, F. Guimbretiere, P. Baudisch, and M. Smith, Stitching, Proceedings of the working conference on Advanced visual interfaces - AVI '04, (2004), pp. 23-31. https://doi.org/10.1145/989863.989866 